\title{
Escribiendo documentos con contenido estadístico
}

\author{
Writing Papers with Statistical Contents
}

Andrés Gutiérrez ${ }^{\mathrm{a}}$

hugogutierrez@usantotomas.edu.co

\section{Resumen}

La Facultad de Estadística de la Universidad Santo Tomás, primer claustro universitario de Colombia, ha lanzado su revista de publicación semestral llamada Comunicaciones en Estadística. En este primer artículo, el comité editorial intenta unificar la forma de presentación de los artículos sometidos a nuestra publicación. Esta es una guía que los autores deben considerar, mas no implica que los autores no puedan hacer variaciones en el contenido de los artículos.

Palabras clave: artículos, unificación de estilo.

\begin{abstract}
The Statistics Faculty in the Universidad Santo Tomás de Colombia has just issued its new semestral publication named Comunicaciones en Estadística. This is the first paper of the journal in which the editorial committee attempts to unify the presentation of the papers that are submitted. It is recalled that this is just a guide that authors could considered but it is clear that variations of the presentation are allowed.
\end{abstract}

Key words: papers, style unification.

\section{Introducción}

La unificación de la forma de presentación de artículos sometidos a esta publicación se basa en un conjunto de conceptos que integran la generalidad de un artículo. Estos conceptos son interpretados de diferentes formas, (todas válidas), por los autores de un artículo. Para homologar la presentación de los artículos se ha propuesto una definición particular, dada por el comité editorial de la revista, de cada concepto, de manera que sirva de guía al autor para que al momento de someter un manuscrito a nuestra revista el proceso de arbitraje sea lo más diáfano posible.

${ }^{\mathrm{a}}$ Director. Centro de Investigaciones y Estudios Estadísticos (CIEES) 


\section{Sugerencias de escritura}

En esta sección, siguiendo las recomendaciones del comité editorial, el autor da una clara descripción, definición y ejemplos de los conceptos más importantes que integran el cuerpo de un artículo. Nótese que cada una de los comentarios dados en los conceptos corresponden a abstracciones propias y no intentan ser un patrón obligado en la escritura de los artículos.

\subsection{Título}

Dada la naturaleza de nuestra publicación y el público objetivo al que pretendemos llegar, es necesario decir que no todos los artículos serán leídos en su totalidad. Este es un hecho que no se puede esconder y que depende en un buen porcentaje del título que lleve el artículo. Si bien no todos los artículos son leídos, lo mismo no sucede con los títulos de los artículos; todos lo títulos son leídos y, por consiguiente, cada una de las palabras que conforman el título del artículo deben ser escogidas con mucho cuidado.

La mayoría de editores de publicaciones seriadas estaría de acuerdo en admitir que un título debe contener la menor cantidad de palabras que, así mismo, logren describir el contenido del artículo. Un título mal puesto, tiene el poder de hacer que el artículo nunca sea leído por la audiencia correcta.

Es necesario que el autor tenga en cuenta que nuestra publicación necesita títulos específicos, cuya sintaxis sea muy elaborada. El autor debe desligarse de todos los preconceptos arraigados por el marketing y someterse al carácter científico de la publicación. Nuestra obligación no es vender; es comunicar nuestras ideas al público indicado. El título es un rótulo, no es una oración y por tanto la estructura clásica de Sujeto + Verbo + Predicado debe desaparecer en este aparte. El título es un conjunto de palabras, mucho más simple y corto que una oración. Al poner el título al artículo, el autor debe preguntarse: ¿cómo buscaría esta clase de información en un índice internacional?

\subsection{Autores}

En el ámbito de la publicación científica abundan los malos entendidos al momento de citar, ordenar e incluir a las personas que, de una u otra forma, han colaborado en la redacción y publicación del artículo. Existe una marcada diferencia entre colaborador y autor: el autor debe colaborar en la producción del artículo; sin embargo, no todo colaborador debe ser incluido en la lista de autores. Esta lista deberá incluir a aquellas personas que han contribuido activamente en el diseño y ejecución de las temáticas del artículo, sin las cuales no se hubiera llevado a buen fin la escritura final del material.

Algunas revistas europeas requieren que la aparición de los autores esté dada siguiendo el orden alfabético. De esta manera, se evade el debate de cuál autor ha 
contribuido más al desarrollo del artículo. Por otra parte, es común definir que el primer autor sea el progenitor del trabajo que se quiere reportar.

Comunicaciones en Estadística asume que todos los autores han contribuido a la publicación del artículo de igual manera y deja libre la escogencia del orden de aparición de los autores en el artículo. El comité editorial de la presente publicación no aceptará títulos académicos seguidos del nombre de los autores. Estamos seguros de que las contribuciones a la ciencia no están ligadas a un título de posgrado.

\subsection{Resumen}

El autor de un artículo debe estar en la capacidad de escribir una exposición de la totalidad del tema en menos de 250 palabras. Aunque no es una tarea fácil, se recomienda omitir cualquier detalle experimental, referencias bibliográficas previas (sin importar el valor crítico que tengan) y omitir cualquier alusión extensa al desarrollo del saber estadístico. Es deber del autor plasmar en el resumen una corta descripción del problema tratado y de la solución encontrada.

Un resumen bien escrito abre la puerta al lector ávido de información, permitiendo la identificación del contenido básico y determinando la relevancia de la lectura de la totalidad del artículo.

Un resumen debe cumplir con los siguientes objetivos:

1. Exponer los principales objetivos, explicando los alcances de la investigación.

2. Describir la metodología empleada en la solución del problema de investigación.

3. Resumir los resultados.

4. Plantear las conclusiones encontradas ${ }^{1}$.

Usualmente, un buen resumen es un indicador de un buen artículo, y un resumen mal redactado es emisario de un largo camino de correcciones. Comunicaciones en Estadística recomienda que los autores examinen cada palabra con sumo cuidado. Si el resumen puede ser redactado utilizando 100 palabras, no use 200. En este punto, cabe recordar la anécdota de un científico que propuso una densa teoría física. Él empezó a escribir el resumen y a darle los debidos retoques eliminando palabras innecesarias. Finalmente, escribió el resumen más corto del campo científico

$$
e=m c^{2} .
$$

El comité editorial está interesado en el uso de una sintaxis clara que utilice las palabras apropiadas, que permita mostrar que el resumen se aleja de todo tipo

\footnotetext{
${ }^{1}$ La esencia del artículo deben ser las conclusiones. Por tanto, estas deben aparecer en el resumen, en la introducción y en la discusión del manuscrito.
} 
de construcciones gramaticales tediosas que impidan la clara identificación de los objetivos del artículo.

Las palabras clave deben aportar la conveniencia necesaria de un servicio de indexación. Una costumbre frecuente es incluir palabras que ya están presentes en el título del artículo; lo anterior lleva a redundancias en el momento de la indexación. El comité editorial de Comunicaciones en estadística ha acordado que la aparición de las palabras clave sea en orden alfabético.

\subsection{Introducción}

La introducción es el camino preliminar al desarrollo del trabajo que se quiere publicar. Debe contener todos los antecedentes de la investigación y suplir al lector de suficiente información para que él esté en la capacidad de evaluar y entender los resultados del estudio. Una buena introducción debe contener los siguientes aspectos:

- Presentar con total claridad la naturaleza y el alcance del problema investigado.

- Hacer una revisión de la literatura pertinente para orientar al lector.

- Exponer el método de investigación, las razones por la que es necesaria la investigación y la motivación de los métodos particulares usados en el desarrollo del trabajo.

- Plantear los principales resultados de la investigación. El autor no debe poner al lector en suspenso; en vez de eso, el autor debe permitir que el lector siga el curso de la evidencia.

La introducción es el lugar correcto para definir cualquier término especializado o abreviación que será usada en apartes posteriores del artículo.

\subsection{Resultados}

Esta sección tiene dos grande núcleos; el primero pretende dar a conocer al público una descripción acerca de los experimentos, simulaciones, estudios computacionales, teóricos y sin entrar en una discusión profunda sobre la naturaleza per se de los mismos; el segundo núcleo se refiere a la presentación de los datos como sustento principal de las conclusiones del artículo.

En la sección de los resultados se deben presentar datos que involucren procesos decisorios y que sean evidencia fundamental en contra o a favor de la técnica estadística utilizada. Se debe evitar la repetitividad al presentar los datos. En esta sección el lector requiere información, no simplemente hileras y montones de datos. 
Por ejemplo, si al realizar una simulación de Monte Carlo para contrastar la eficiencia relativa de varios estimadores, resulta que el sesgo relativo es despreciable para cada uno de ellos; entonces, será un desperdicio de papel y tinta la inclusión de una tabla con valores muy cercanos a cero. Para evitar esto, el autor debe resumir estos hallazgos en una simple frase.

Aunque la sección de los resultados es la parte más importante del artículo, es a veces la más corta. Esto no tiene consecuencia alguna sobre la buena lectura del manuscrito sí esta sección está precedida por una explicación clara y detallada del marco teórico y de las técnicas utilizadas. Cuando existen figuras (gráficas o tablas) que soportan los hallazgos fundamentales del artículo, es muy posible que el autor cometa el pecado de la redundancia.

Por ejemplo, si la figura se refiere a la siguiente tabla:

\begin{tabular}{|c|c|c|}
\hline & Machos & Hembras \\
\hline Media muestral & 4.762 & 2.396 \\
\hline Diferencia & \multicolumn{2}{|c|}{2.366} \\
\hline Valor P & \multicolumn{2}{|c|}{0.013} \\
\hline
\end{tabular}

Tabla 1: Diferencias entre machos y hembras

Se cae en este error al presentarla de la siguiente manera:

De la Tabla 1, se puede concluir claramente que la diferencia de medias es estadísticamente significativa.

La manera correcta de informar al lector sobre este hallazgo está dada mediante la siguiente afirmación:

La diferencia de medias es estadísticamente significativa (Tabla 1).

\subsection{Discusión}

Es la sección que resulta más difícil de escribir. Muchos editores de revistas seriadas rechazan un artículo para publicación porque no adjunta una buena discusión, incluso si los resultados del artículo son válidos e interesantes. Los componentes de una discusión son los siguientes:

- Tratar de presentar los principios, relaciones y generalizaciones de la sección de los resultados. Precisamente, en una buena discusión se discute, no se resume.

- Puntualizar cualquier excepción.

- Mostrar cómo los resultados e interpretaciones concuerdan (o divergen) con los resultados e interpretaciones de anteriores publicaciones. 
- Discutir las implicaciones teóricas y prácticas del trabajo realizado.

- Exponer las conclusiones tan claramente como sea posible.

\subsection{Agradecimientos}

En honor a la verdad, agradecer no es tarea fácil. En algunas ocasiones, simplemente; en algunas otras, nos desbordamos en agradecimientos. Lo cierto es que en cuestión de artículos publicados, el autor debe agradecer especificamente a aquellas personas que colaboraron en el desarrollo de la investigación. El agradecimiento debe ser, también, específico y concreto, mencionando el motivo por el cual el autor agradece. Por ejemplo:

El autor agradece a Pepe Pérez por la asistencia computacional y a Juan Rodríguez por la ayuda prestada en la demostración de los teoremas.

Si el autor ha recibido algún tipo de ayuda por parte de una institución gubernamental o privada, o de algún grupo de investigación, se debe mencionar en el manuscrito.

Nótese la necesidad de ser específico en cuanto a los agradecimientos, porque la persona que ha colaborado con el autor puede estar en desacuerdo con lo expuesto en el artículo. En esta situación, agradecer de manera general indicaría un parte de responsabilidad de parte del colaborador. Una vez más, como se busca la máxima claridad posible, el autor deberá suprimir el verbo desear en los agradecimientos. En vez de escribir:

Yo deseo agradecer a Pedro Pérez.

El autor deberá escribir:

Yo agradezco a Pedro Pérez.

Es evidente que la primera opción puede ser entendida por algunos lectores como que el autor desearía agradecer a Pedro Pérez, pero no lo ha hecho porque Pedro Pérez no ha ayudado lo suficiente.

\subsection{Referencias bibliográficas}

Todas las referencias citadas en el artículo deben aparecer en el cuerpo del documento. Sólo se deberán citar aquellas que han tenido un impacto significativo en el desarrollo de la publicación. 
Comunicaciones en Estadística utiliza el sistema de apellido y año, más conocido como el sistema Harvard. De esta manera, el apellido del autor aparece seguido del año de la publicación. Por ejemplo: « (De Castro 2003) ». Si el autor tiene dos o más publicaciones en el mismo año, entonces se agregará una letra minúscula en orden alfabético después del año. Por ejemplo « (De Castro 2007a) » y/o « (De Castro 2007b) ».

En el caso de que el artículo haya sido escrito por tres o más autores, se escribirá la referencia completa sólo la primera vez que sea citada. Por ejemplo « (Pinto, Martínez y Smith 2008) ». Las próximas citaciones de la misma referencia se simplificarán usando la abreviatura latina et al, de esta manera, « (Pinto et. al. 2008)».

\subsection{Otras}

Dado que Comunicaciones en Estadística intenta llegar a una audiencia específica, el lenguaje que se utilizará en la mayoría de los artículos será técnico, seguido por expresiones matemáticas y estadísticas. Existen varias acotaciones que el comité editorial tiene al respecto.

\subsubsection{Figuras}

Las tablas y gráficos de los artículos sometidos y publicados en Comunicaciones en Estadística deberán tener un rótulo general. La leyenda de la figura (tablas o gráficas) es como el título del artículo y por lo tanto se deben seguir las mismas pautas expuestas en las secciones anteriores. Por ejemplo:

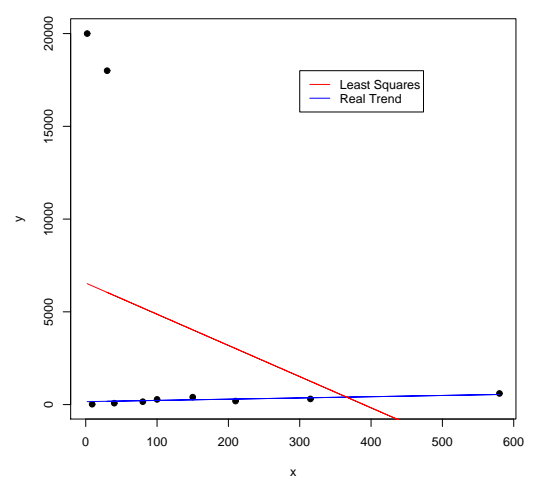

Figura 1: Dos posibles líneas de regresión

Si el autor decide insertar una gráfica o una tabla está en la obligación de comentarla en el cuerpo del documento inmediatamente después de su aparición. 


\subsubsection{Fórmulas}

El autor de un texto estadístico debe utilizar los signos de puntuación en las expresiones matemáticas. Nótese que la utilización de fórmulas no es más que una abreviación de una frase. Así que, como tal, es necesario utilizar la coma, el punto y coma y el punto después de cada fórmula, según sea el caso.

Cuando las expresiones matemáticas hagan parte de un párrafo y no se encuentren centradas o numeradas, el autor deberá tratar de que la fórmula no corrompa la forma visual del artículo. Por ejemplo, al escribir la fracción $\frac{a+b}{c}$ la visualización del artículo se ve alterada. La forma correcta de escritura es $(a+b) / c$ o en su defecto $\frac{a+b}{c}$.

\subsubsection{Tiempo gramatical}

Un artículo estadístico maneja diferentes tiempos gramaticales. El resumen del artículo, por lo general, se encuentra en pasado porque se hace referencia a resultados que ya se encontraron. La introducción y la discusión, se escriben por lo general en presente porque enfatizan conocimientos establecidos de antemano y discuten posibles formas de acceder a la investigación. El cuerpo del documento y la explicación de la teoría pueden estar en presente o en pasado, según sea el caso.

\subsubsection{Valores estadísticos}

En un mismo párrafo es posible encontrar una mezcla del pasado y el presente en algunas excepciones. Una de esas excepciones es cuando el autor hace referencia a valores surgidos de análisis estadísticos que deben estar en tiempo presente, incluso cuando la frase esté construida en tiempo pasado. Por ejemplo:

Estos valores son significativamente más grandes que los de las hembras de la misma edad, lo que indica que los machos crecieron más rápidamente.

\subsubsection{Voz activa y voz pasiva}

Un error común en el que el principiante incurre es en usar la voz pasiva. De hecho, no existe ninguna razón para no usar la voz activa en la redacción del documento. Como resultado el estadístico escribe, la mayoría de las veces frases como «se encontró que» en vez de «yo encontré que»o «hemos encontrado que».

$\mathrm{El}$ autor debe renunciar a la falsa modestia de las anteriores generaciones. No hay que temer a enunciar el nombre de quién ha ejecutado una acción. Sin embargo, no es elegante que en un artículo escrito por un solo autor se encuentre un pronombre plural. Por ejemplo, «hemos concluido que». 


\section{Artículos de revisión}

Una revisión no es un artículo original. Sin embargo, el propósito de un artículo con estas características es revisar la literatura que se ha publicado con antelación y ponerla en perspectiva.

Esta clase de documentos tiende a tener una extensión bastante mayor a la usual. De hecho se habla de que la extensión de una revisión oscila entre 10 y 50 páginas. El objeto de una revisión debe ser general, aunque no se debe limitar a meras anotaciones bibliográficas. Un buen artículo de revisión debe ofrecer una evaluación crítica de la literatura publicada y proveer conclusiones acerca de esa literatura.

El contorno del artículo debe ser preparado cuidadosamente. De esta manera, habrá un orden implícito en la escritura y redacción del documento. Un artículo de revisión, no necesariamente debe estar escrito en orden cronológico. De acuerdo al estado del arte, las fechas de las técnicas o metodologías estadísticas pueden aparecer irrespetando la cronología. Sin embargo, cuando el autor desea exponer un tema que no ha sido revisado previamente, el manuscrito debe contener una fuerte base de fundamentos históricos. Si el tema de discusión ya ha sido revisado, el punto de partida del artículo debe ser la última revisión del tema.

Nótese que la audiencia de un artículo de revisión es mucho más general que la audiencia de un artículo especializado. De esta forma, el artículo de revisión se hace mucho más valioso en los procesos de enseñanza estadística, máxime cuando las conclusiones del mismo deben ser claras y deben invitar a la disertación de los fundamentos, aplicaciones e implicaciones del tema discutido.

\section{Escribiendo el manuscrito}

Al finalizar la etapa de experimentación, desarrollo teórico y aplicación práctica, sigue la escritura del documento. Algunos autores caen en el error de creer que como el documento es parte de un trabajo estadístico científico, entonces los datos hablarán por sí mismos y el editor debe publicar el artículo. Lo cierto es que la anterior clase de pensamiento es totalmente falsa. El consejo del comité editorial a determinado que si el autor en verdad desea publicar su material en Comunicaciones en Estadística debe estar seguro de que el manuscrito que se ha entregado esté libre de errores ortográficos y tipográficos, más aún, con una sintaxis clara y explícita. Para lo anterior, es recomendable que el autor lea el manuscrito antes de enviarlo al comité editorial. De otra parte, es conveniente que la lectura sea también realizada por un colega ajeno a la investigación. El anterior proceso asegurará un arbitraje enfocado y objetivo y, por qué no decirlo, una probabilidad alta de aceptación.

\section{Recuerde que la fácil lectura requiere un gran esfuerzo en la escritura.}




\section{Agradecimientos}

De antemano agradezco a los autores que formarán parte de esta gran familia, por la confianza depositada en nosotros al entregarnos cada uno de los artículos sometidos. El mensaje para ellos es que aseguraremos la excelencia en el arbitraje, así como también en la publicación y en el trato editorial.

Aprovecho este espacio para agradecer al Dr. Sander Rangel, decano de la Facultad de Estadística, por su visión estratégica acompañada de una excelente gestión administrativa, y al Dr. Camilo Cubides, por su colaboración y disposición en la edición de la plantilla de $\mathrm{LT}_{\mathrm{E} X}$.

\section{Referencias}

De Castro, R. (2003), El universo LT $_{E} X$, segunda edn, Unibiblos, Universidad Nacional de Colombia, Bogotá, Colombia.

Knuth, D. E. (1986), The TEXbook, Computers and Typesetting, pub-AW, pubAW:adr.

Lamport, L. (1994), LATEX: A Document Preparation System, second edn, AddisonWesley Professional. 